\title{
Pomalidomide: when expectations are understated
}

\author{
"The treatment with pomalidomide plus low-dose dexamethasone and \\ the latest advances of therapies in relapsed-refractory multiple \\ myeloma are analyzed in the field of practice setting, in order to \\ provide insights into possible approaches to the treatment of \\ relapsed-refractory multiple myeloma."
}

\section{Francesco Di Raimondo*,1 \& Concetta Conticello'}

First draft submitted: 2 August 2016; Accepted for publication: 19 December 2016; Published online: 24 January 2017

Multiple myeloma (MM) largely remains an incurable disease. Progressively acquired drug resistance is a major reason for the limited benefit of most MM therapies. In 2004, Kumar et al. [1] conducted a study on outcomes in relapsed MM. The study revealed decreasing response duration with increasing number of salvage regimens, likely reflecting acquired drug resistance. More recently, the International Myeloma Working Group [2] investigated outcomes in patients with relapsed-refractory MM (RRMM). The median overall survival and event-free survival from T0 were 9 and 5 months, respectively. The study has confirmed the poor outcome, once patients become refractory to current treatments. Although MM remains a complex and challenging disease, in recent years the management of this disease has benefited substantially from the introduction of new drugs such as proteasome inhibitors and immunomodulatory drugs (IMiDs). These agents exert their effects through several mechanisms including direct cytotoxicity, antiangiogenic effects, inhibition of bone resorption and activation of antitumor immunity.
Among IMiDs, pomalidomide seems to have a more potent antimyeloma activity in RRMM patients, and a similar favorable safety profile compared with thalidomide and lenalidomide [3].

In Phase I-II studies, pomalidomide plus low-dose dexamethasone (Poma + LoDEX) demonstrated activity in MM patients refractory to both bortezomib and other IMiDs [4-6]. The pivotal, multicenter, open-label, randomized Phase III trial (MM-003) [7] compared Poma + LoDEX with high-dose dexamethasone in previously treated adult patients with RRMM who had received at least two prior treatment regimens, including both lenalidomide and bortezomib, and had demonstrated disease progression on the last therapy. In this study on overtreated patients, overall survival - possibly underestimated because of the crossover from high-dose dexamethasone to Poma + LoDEX arm - was 12.7 months, whereas progressionfree survival was 4 months, with a median follow-up of 10 months. Based on the results of this Phase III trial, EMA granted accelerated approval of pomalidomide, which is now considered a new effective strategy for RRMM patients [8].

'Department of Clinical \& Molecular Biomedicine, Haematology Section, University of Catania, Catania, Italy

*Author for correspondence: diraimon@unict.it

\section{KEYWORDS}

- drug resistance

- immunomodulatory drugs

- multiple myeloma • pomalidomide
Future $\mathrm{fSg}$ 
Interestingly, in these clinical trials time to progression achieved with Poma + LoDEX in responding patients was superior to time to progression achieved with the immediately prior treatment: this finding suggests that pomalidomide is able to change the natural course of disease.

Recently, more evidence has been accumulated on the efficacy of pomalidomide in patients that have relapsed after lenalidomide or proteasome inhibitors treatments $[9,10]$. However, 'field-practice' information is necessary for a more grounded evaluation of the benefits of pomalidomide. Indeed, field-practice experiences, including case reports, are important since they complement evidence derived from clinical trials in unselected patients often presenting multiple comorbidities or challenging management [11,12].
In this supplement, we present two cases of MM patients refractory to previous treatments including bortezomib and lenalidomide. The treatment with Poma + LoDEX and the latest advances of therapies in RRMM are analyzed in the field of practice setting, in order to provide insights into possible approaches to the treatment of RRMM.

Financial \& competing interests disclosure The authors have no relevant affiliations or financial involvement with any organization or entity with a financial interest in or financial conflict with the subject matter or materials discussed in the manuscript. This includes employment, consultancies, honoraria, stock ownership or options, expert testimony, grants or patents received or pending, or royalties.

Editorial assistance for the preparation of this manuscript was provided by Luca Giacomelli on behalf of Content Ed Net. This assistance was funded by Celgene.

\section{References}

1 Kumar SK, Therneau TM, Gertz MA et al. Clinical course of patients with relapsed multiple myeloma. Mayo Clin. Proc. 79(7), 867-874 (2004).

2 Kumar SK, Lee JH, Lahuerta JJ et al. Risk of progression and survival in multiple myeloma relapsing after therapy with IMiDs and bortezomib: a multicenter international myeloma working group study. Leukemia 26(1), 149-157 (2012).

3 Offidani M, Corvatta L, Caraffa P et al. Pomalidomide for the treatment of relapsedrefractory multiple myeloma: a review of biological and clinical data. Expert Rev. Anticancer Ther. 14(5), 499-510 (2014).

4 Schey SA, Fields P, Bartlett JB et al. Phase I study of an immunomodulatory thalidomide analog, CC-4047, in relapsed or refractory multiple myeloma. J. Clin. Oncol. 22(16), 3269-3276 (2004).

5 Richardson PG, Siegel D, Baz R et al. Phase I study of pomalidomide MTD, safety, and efficacy in patients with refractory multiple myeloma who have received lenalidomide and bortezomib. Blood 121, 1961-1967 (2013).

6 Richardson PG, Siegel DS, Vij R et al. Pomalidomide alone or in combination with low-dose dexamethasone in relapsed and refractory multiple myeloma: a randomized Phase II study. Blood 123, 1826-1832 (2014).

7 San Miguel J, Weisel K, Moreau P et al. Pomalidomide plus low-dose dexamethasone versus high-dose dexamethasone alone for patients with relapsed and refractory multiple myeloma (MM-003): a randomised, open-label, Phase III trial. Lancet Oncol. 14(11), 1055-66 (2013).

8 Hanaizi Z, Flores B, Hemmings R et al. The European medicines agency review of pomalidomide in combination with low-dose dexamethasone for the treatment of adult patients with multiple myeloma: summary of the scientific assessment of the committee for medicinal products for human use. Oncologist 20(3), 329-334 (2015).
9 Dimopoulos MA, Palumbo A, Corradini P et al. Safety and efficacy of pomalidomide plus low-dose dexamethasone in STRATUSTM (MM-010): a Phase IIIb study in refractory multiple myeloma. Blood 128(4), 497-503 (2016).

10 San Miguel JF, Weisel KC, Song KW et al. Impact of prior treatment and depth of response on survival in MM-003, a randomized Phase III study comparing pomalidomide plus low-dose dexamethasone versus high-dose dexamethasone in relapsed/ refractory multiple myeloma. Haematologica 100(10), 334-339 (2015).

11 Rizzo M, Cartenì G, Pappagallo G. We need both randomized trials and real-world data: the example of everolimus as second-line therapy for mRCC. Future Oncol. 10, 1893-1896 (2014).

12 Barni S. Eribulin in 'field practice': an overview of the Italian experience. Future Oncol. 11(Suppl. 15), 1-2 (2015). 